\title{
Projective synchronization control of fractional-order chaotic system
}

\section{Fandi Zhang ${ }^{\text {a }}$}

\author{
School of Mathematic and statistics, Tianshui Normal University, Tianshui 741001, China \\ azhangfand123@163.com
}

\begin{abstract}
This paper investigates projective synchronization of fractional order chaotic systems with different orders. In order to get the projective synchronization of fractional order chaotic systems with different orders, a scheme is proposed and effective controllers for synchronization are designed. Numerical simulations on examples are presented to show the effectiveness of the proposed control strategy.
\end{abstract}

Keywords: Fractional-order system; Projective synchronization; Stability theory

\section{Introduction}

In the past decades, synchronization has gained attention due to its potential applications in various fields [1-3]. Moreover, different synchronization types have been presented in chaotic systems, such as phase synchronization [4], anti-synchronization [5], projective synchronization [6] and so on. Meanwhile, effective control methods have been proposed for various synchronization types. As we known, many researchers pay their attention to the study of fractional-order chaos synchronization [7]. Fractional calculus is the generalization of the conventional calculus. It has been found that the fractional calculus has many applications in secure communication, complex network and so on. Rich dynamical behaviours exist in fractioanl-order system. That is to say, it is very interesting to study the projective synchronization between fractional-order systems. In this paper. We focus on demonstrate the validity of the above proposed methods for the projective synchronization on the fractional-order system.

\section{Fractional-order definition}

The fractional order is the extending concept of integer-order operator, which can be described as following:

$$
{ }_{a} D_{t}^{q}=\left\{\begin{array}{lc}
\frac{d^{q}}{d t^{q}} & R(q)>0 \\
1 & R(q)=0 \\
\int_{a}^{t}(d \tau)^{-q} & R(q)<0
\end{array}\right.
$$

where $q$ is the fractional order which can be a complex number. The Riemann-Liouville fractional derivative of order $\alpha \geq 0$ of the function $f(t)$ is defined as

$$
J^{\alpha} f(t)=\frac{1}{\Gamma(\alpha)} \int_{0}^{t}(t-\tau) f(\tau) d \tau, \quad t>0
$$

Caupto definition of the fractional derivative of the function $f(t)$ is defined as:

$$
D_{*}^{\alpha} f(t)=\frac{1}{\Gamma(m-\alpha)} \int_{0}^{t} \frac{f^{(m)}(\tau)}{(t-\tau)^{\alpha-m+1}} f(\tau) d \tau, \quad t>0 .
$$

Formula (2) will be used in this paper where $\alpha$ is the fractional order. 


\section{Synchronization scheme}

A fractional order hyperchaotic drive system is given by

$$
D^{\alpha} X=A X+F(X)
$$

similarly, a response system is given as follows,

$$
D^{\beta} Y=B Y+G(Y)+U(X, Y, t)
$$

where $X=\left(x_{1}, x_{2}, \ldots x_{n}\right)^{T}, Y=\left(y_{1}, y_{2}, \ldots y_{n}\right)^{T}$ denote state vectors, $F: R^{n} \rightarrow R^{n}, G: R^{n} \rightarrow R^{n}$ are continuous vector functions, $U(X, Y, t)$ is a controller which will be designed.

We take hyper-chaotic fractional-order Lorenz system is the drive system, it can be described as below:

$$
\left\{\begin{array}{l}
D^{\alpha} x_{1}=a\left(x_{2}-x_{1}\right)+x_{4} \\
D^{\alpha} x_{2}=b x_{1}-x_{2}-x_{1} x_{3} \\
D^{\alpha} x_{3}=x_{1} x_{2}-c x_{3} \\
D^{\alpha} x_{4}=-x_{2} x_{3}+d x_{4},
\end{array}\right.
$$

when $\alpha=0.96$ and $(d, h, f, r)=(10,8 / 3,28,-1)$, the fractional-order Lorenz hyper-chaotic attractor is shown in Fig.1.

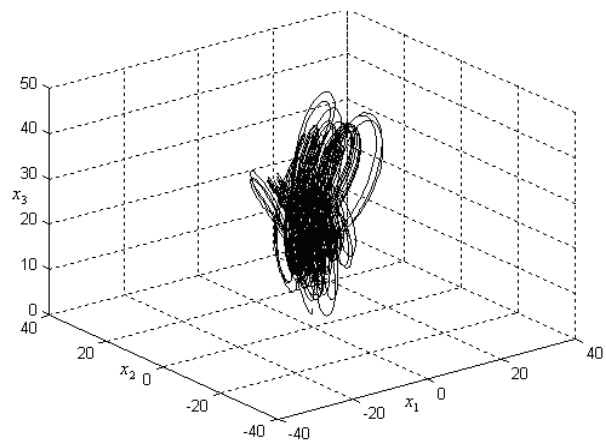

Fig.1.The attractor of Lorenz Fractional-order system with $q=0.96$

$$
\left\{\begin{array}{l}
D^{\beta} y_{1}=a_{1}\left(y_{2}-y_{1}\right)+y_{4} \\
D^{\beta} y_{2}=d_{1} y_{1}-y_{1} y_{3}+c y_{2} \\
D^{\beta} y_{3}=-b_{1} y_{3}+y_{1} y_{2} \\
D^{\beta} y_{4}=y_{3} y_{2}+0.5 y_{4},
\end{array}\right.
$$

We consider system (7) as the response system, the attractor is shown in Fig.2

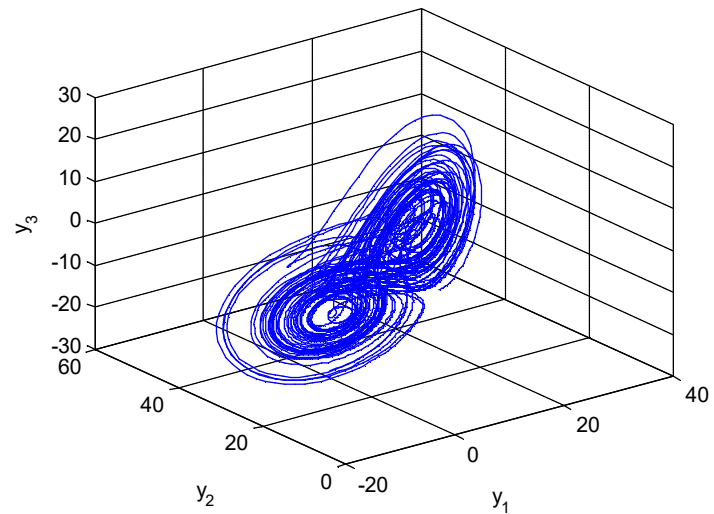

Fig.2.The attractor of Chen fractional-order hyperchaotic system with $q=0.99$

Based on the aforementioned analysis, the systems (6) and (7) can be rewritten as following: 


$$
\begin{aligned}
& {\left[\begin{array}{l}
D^{\alpha} x_{1} \\
D^{\alpha} x_{2} \\
D^{\alpha} x_{3} \\
D^{\alpha} x_{4}
\end{array}\right]=\left[\begin{array}{cccc}
-a & a & 0 & 1 \\
b & -1 & 0 & 0 \\
0 & 0 & -c & 0 \\
0 & 0 & 0 & d
\end{array}\right]\left[\begin{array}{l}
x_{1} \\
x_{2} \\
x_{3} \\
x_{4}
\end{array}\right]+\left[\begin{array}{l}
0 \\
0 \\
1 \\
0
\end{array}\right]\left(x_{1} x_{2}\right)+\left[\begin{array}{c}
0 \\
-1 \\
0 \\
0
\end{array}\right]\left(x_{1} x_{3}\right)+\left[\begin{array}{c}
0 \\
0 \\
0 \\
-1
\end{array}\right]\left(x_{2} x_{3}\right)} \\
& {\left[\begin{array}{l}
D^{\beta} y_{1} \\
D^{\beta} y_{2} \\
D^{\beta} y_{3} \\
D^{\beta} y_{4}
\end{array}\right]=\left[\begin{array}{cccc}
-a_{1} & a_{1} & 0 & 0 \\
d_{1} & 0 & c_{1} & 0 \\
0 & 0 & -b_{1} & 0 \\
0 & 0 & 0 & r_{1}
\end{array}\right]\left[\begin{array}{l}
y_{1} \\
y_{2} \\
y_{3} \\
y_{4}
\end{array}\right]+\left[\begin{array}{c}
0 \\
-1 \\
0 \\
0
\end{array}\right]\left(y_{1} y_{3}\right)+\left[\begin{array}{l}
0 \\
0 \\
1 \\
0
\end{array}\right]\left(y_{1} y_{2}\right)+\left[\begin{array}{l}
0 \\
0 \\
0 \\
1
\end{array}\right]\left(y_{3} y_{2}\right)+\left[\begin{array}{l}
u_{1} \\
u_{2} \\
u_{3} \\
u_{4}
\end{array}\right]}
\end{aligned}
$$

According to the synchronization scheme presented in the previous discussion, we choose scaling matrix $\Lambda=\operatorname{diag}\left(\lambda_{1}, \lambda_{2}, \ldots \lambda_{n}\right)$, where $\lambda_{i}$ are the real constant. The error vector can be defined as

$$
\left\{\begin{array}{l}
e_{1}(t)=y_{1}-\lambda_{1} x_{1} \\
e_{2}(t)=y_{2}-\lambda_{2} x_{2} \\
e_{3}(t)=y_{3}-\lambda_{3} x_{3} \\
e_{4}(t)=y_{4}-\lambda_{4} x_{4}
\end{array}\right.
$$

The following criterion is proposed to ensure that the response system (10) effectively synchronizes the drive system (10) up to a scaling matrix.

Theorem 3.2. For a given constant scaling matrix and any initial conditions, modified projective synchronization between systems (10) and (11) will occur by the following adaptive controllers:

$$
\left\{\begin{array}{l}
\left.u_{1}(t)=D^{-(\alpha-\beta)}\left[\lambda_{1}\left(\left(a_{1}-a\right) x_{1}+\left(a-a_{1}\right) x_{2}\right)+x_{4}\right)-K_{1} E\right] \\
u_{2}(t)=\left(D^{-(\alpha-\beta)}-I\right)\left(-y_{1} y_{3}\right)+D^{-(\alpha-\beta)}\left[\lambda_{2}\left(\left(d-b_{1}\right) x_{1}-c_{1} x_{3}-x_{2}\right)+y_{1} y_{3}-x_{1} x_{3}-K_{2} E\right] \\
\left.u_{3}(t)=\left(D^{-(\alpha-\beta)}-I\right)\left(y_{1} y_{2}\right)+D^{-(\alpha-\beta)}\left[\lambda_{3}\left(\left(b_{1}-c\right) x_{3}-y_{1} y_{2}\right)\right)+\left(x_{1} x_{2}\right)-K_{3} E\right] \\
u_{4}(t)=\left(D^{-(\alpha-\beta)}-I\right)\left(y_{3} y_{2}\right)+D^{-(\alpha-\beta)}\left[\lambda_{4}\left(d-r_{1}\right) x_{4}-y_{2} y_{3}-x_{2} x_{3}-K_{4} E\right]
\end{array}\right.
$$

where $K_{i}(i=1,2,3,4)$ denote the $i$ th line of matrix $K . \lambda_{i}(i=1,2,3,4)$ is scaling factor.

Then the error system is calculated as follows

$$
D^{\alpha} E=(B-K) E=\left[\begin{array}{cccc}
-1 & 0 & 0 & 0 \\
0 & -1 & 0 & 0 \\
0 & 0 & -5 & 0 \\
0 & 0 & 0 & -0.5
\end{array}\right] \times\left[\begin{array}{c}
e_{1} \\
e_{2} \\
e_{3} \\
e_{4}
\end{array}\right]
$$

The eigenvalues of the matrix $B-K$ satisfy $\left|\arg \left(\lambda_{i}\right)\right|>\frac{\alpha_{i}}{2} \pi=\frac{0.96 \pi}{2}$. According to the stability theorems of the fractional-order system, the error system is asymptotically stable and the synchronization is guaranteed.

We choose the scaling factor as $\lambda_{1}=2, \lambda_{2}=-1, \lambda_{3}=3, \lambda_{4}=1$. The synchronization errors are shown in Fig.3. In Fig.4, The phase portraits in drive and response system with different initial conditions are presented. 


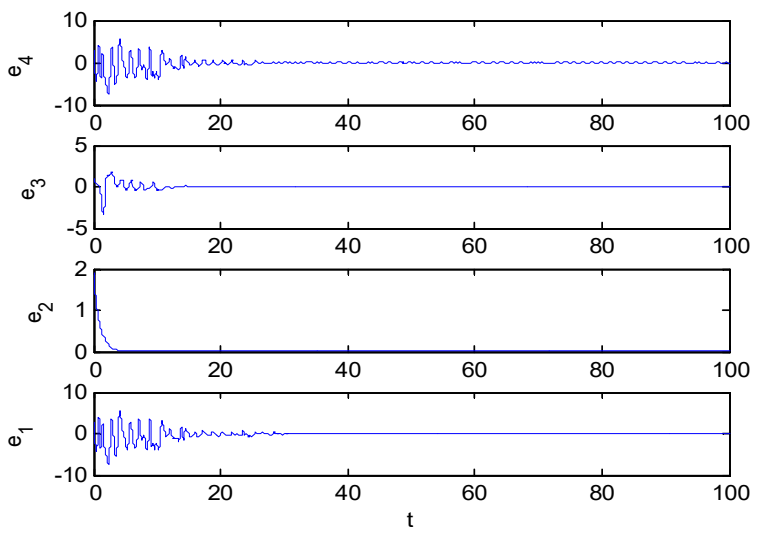

Fig.2. Synchronization errors between systems (6) and (7) with evolving time $t$
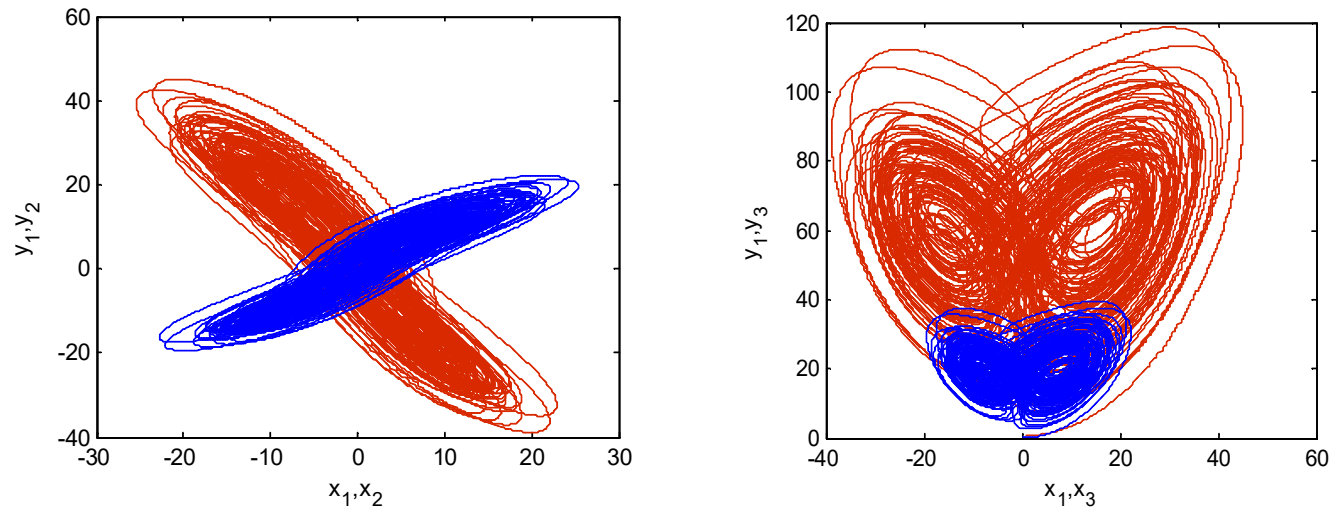

Fig.3. Phase portraits of the drive system (6) and response systems (7)

\section{Conclusion}

In this paper, projective synchronization of fractional-order chaotic system is discussed. Based on the stability theorem of fractional-order systems, projective synchronization of the fractional order chaotic system is achieved. The effectiveness of strategy is validated by numerical simulations.

\section{References}

[1] B. Blasius, A. Huppert, L. Stone, Complex dynamics and phase synchronization in spatially extended ecological systems. Nature, Vol.399(1999),No.2,p.354-359, 1999.

[2] S.H. Chen, J.H. Lu, Parameters identication and synchronization of chaotic systems based upon adaptive control. Physics Letters A, Vol.299(2002), No.2,p.296-303.

[3] K. Diethelm, N.J. Ford, A.D. Freed. A predictor- corrector approach for the numerical solution of fractional differential equations. Nonlinear Dyn, Vol.(29), No.29, p.3-22.

[4] J.W.Feng, S.H.Chen. Controlling chen hyper-chaotic system. International of Journal Nonlinear Science Numerical Simulations, Vol. 7(2006),No.2,p.369-374.

[5] X.Wu, J. Li, Chaos control and synchronization of a three species food chain model via Holling functional response, Int. J. Comput. Math. Vol.87(2010),no.2 p.199-214.

[6] X.Y.Wang, X.P. Zhang, Modified projective synchronization of fractional-order chaotic systems via active sliding mode control, Nonlinear Dyn, Vol.69 (2012), p.511-517.

[7] R.Mainieri, J. Rehacek, Projective synchronization in three-dimensional chaotic systems, Physic Review Letter, Vol. 82(1999) No.2, p.3042-3045. 